Seyed Siamak Taghavi Larijani and Amin Farrokhabadi*

\title{
A variational approach for predicting initiation of matrix cracking and induced delamination in symmetric composite laminates under in-plane loading
}

https://doi.org/10.1515/secm-2016-0379

Received December 16, 2016; accepted June 6, 2017; previously published online October 17, 2017

\begin{abstract}
This paper discusses the formation of matrix cracking and induced delaminations in a $\left[\phi_{m}^{(2)} / \psi_{n}^{(1)}\right] s$ laminate subject to arbitrary in-plane loading using a variational approach. To this end, the effects of delaminations coming from the tips of transverse cracks in the middle sublaminates are considered to specify the critical crack density points and finally understand which modes of damage are prospects for various lay-ups in the presence of transverse cracks as well as induced delamination. After deriving a very good approximation of the principle of minimum complementary energy by considering complex equations, the stress fields are provided. In this analysis, a unit cell in the ply level of a composite laminate containing both matrix cracking and delamination is considered. Then, the values of the admissible stresses and compliance of cracked laminate are employed to evaluate the energy release rate of each mentioned damage mode. Eventually, the graphs for different lay-ups in such symmetric laminate are drawn, which indicate important points for designers in practical applications. Afterwards, the effects of thickness on the dislocation of critical crack density points are checked. It can be emphasized that the current approach opens a new insight for perceiving the composites' structural behavior in vulnerable positions.
\end{abstract}

Keywords: induced delamination; matrix cracking; transverse cracks; variational approach.

\section{Introduction}

One of the most essential discussions in composites is their innate ability to withstand damage modes. Over the past four decades, a particular focus of discussion for failure composites has been served. There are always

\footnotetext{
*Corresponding author: Amin Farrokhabadi, Department of Mechanical Engineering, Tarbiat Modares University, Tehran 14115-111, Iran, Phone: +982182883938, e-mail: amin-farrokh@modares.ac.ir Seyed Siamak Taghavi Larijani: Department of Aerospace Engineering, Semnan University, Semnan, Iran
}

several possible damages in laminated composites, e.g. matrix cracking [1], delamination [2], etc. Due to the utilization of composites in diverse industries, their effective parameters, properties, and relations with failing factors should be known precisely. For this purpose, researchers have tried to conduct studies about the major damage modes that are occurring in a laminate during the process of loading.

Matrix cracking and induced delamination are the most significant damage modes that often occur in composite laminates under general in-plane loading. The failure processes of composite laminates under general in-plane loading have been permanently seen in diverse situations like matrix cracking and induced delamination. For predicting matrix cracking, distinct models were suggested by researchers [3-7]. All of these models are based on limitations that decrease the accuracy of their calculations. Furthermore, these models are not able to estimate initiation or propagation of damages without considering continuum mechanics thermodynamic properties very well, unless using some assumptions such as changing scales in a unit cell $[8,9]$.

In the unit cell models, the first challenge for many researchers was the estimation of stiffness reduction. Indeed, they have sought to know how matrix cracking in a laminate could decrease the elasticity properties. For this problem, all of the investigations can be divided into three parts: variational approach [10-13], shear-lag method [14-16], and stress transfer method [8, 17-19]. Even though diverse semianalytical or numerical methods can be found in some papers [20,21], this article emphasizes the analytical methods. As a result, depending on the conditions of the problem, for instance, the lay-up or type of loading, the solutions should be suggested.

Hashin [10, 22, 23] employed a variational approach to offer an approximate stress analysis for cross-ply laminates having matrix cracking in the $90^{\circ}$ plies. This method was complemented for the angle-ply lay-ups under general in-plane loading by Vinogradov and Hashin [24]. According to the results presented by Zhang and Minnetyan [25], it can be said that stress-based variational analysis has more rational results in relation to displacement-based variational analysis. In the latest presented studies, the stiffness reductions have been calculated for arbitrary 
lay-ups [11, 12]. In fact, it was attempted to conduct and expand the projects for outer-ply matrix cracking in crossply laminates [26].

Generally, by reviewing stress transfer and shear-lag models, some significant weaknesses can be cited, which should be considered while investigating diverse properties in a laminate. Firstly, the effects of out-plane stress are not considered; however, this stress plays a special role in the stress transfer. Aside from this, the special lay-ups and loading conditions confine results to crossply lay-ups or some off-axis laminates. These objections have been partially decreased in the developed model of shear-lag (McCartney method); however, complex calculations [27] persuade authors not to utilize this method. On the other hand, if special attention is paid to the results of Vinogradov and Hashin [24], it can be said that the process of investigating a laminate is also declined, so that it is decided to employ a variational approach for calculating stiffness analysis.

Due to the importance of the induced delamination mode in the final failure load, some researchers tried to suggest several related analytical or numerical models [28-30]. Nairn and $\mathrm{Hu}$ [31] used the variational approach to predict the initiation and growth of induced delamination by matrix cracking. As a matter of fact, the compliance of cracked laminate in cross-ply lay-ups under specific loading was used to compute the energy release rate for formation matrix cracking in the middle sublaminate and induced delamination; however, it can be seen that in the paper by Hosseini-Toudeshky et al. [8], the energy release rate was based on the stress field of cracked laminate. In other studies [32, 33], Li et al. researched the prediction of continuous matrix cracking; however, their calculations had totally the same boundary and continuity conditions that had been considered by Nairn and $\mathrm{Hu}$ [31], and there is no research for distinct stacking sequences.

By considering the mentioned problem, the present study provides a generalized model that is based on the unit cell method. Using Euler-Lagrange equations in the variational approach, it is attempted to evaluate the energy release rate of induced delamination as well as matrix cracking formation simultaneously for angle-ply laminates. In fact, this paper is a complementary research to Nairn and Hu's study [31], in perspective. Nairn and $\mathrm{Hu}$ tried to compute the critical crack densities by using a dimensionless formulation according to the energy release rates only for cross-ply laminates; however, for angle-ply laminates, this method is impossible because of complex Euler-Lagrange equations that are supposed to be simplified. In this regard, the methods that they employed are no longer unique for this problem. Therefore, the authors attempted to simplify the approach with respect to its principles. In the following, changes that may occur to the critical size of unit cells in the laminates, according to their stacking sequences when there is an equal stress state, are revealed. Using the boundary and continuity conditions, the stress state of the cracked laminate is assessed by considering the fact that these conditions could be arbitrary in regards to the kind of loading; for example, it can be full in-plane stress or only tensile stress in $X$ direction. In the next phase, the effects of thickness in the critical crack density dislocation are estimated. Displacement of this point depends on the size of unit cell investigated. This technique provides a precise lower bound for the stiffness matrix, so that the results of its energy release rates are the most reliable amounts for practical application.

\section{Variational approach under in-plane loading}

\subsection{Perturbation stress field analysis}

Consider an angle-ply $\left[\phi_{m}^{(2)} / \psi_{n}^{(1)}\right] s$ composite laminate that is cracked under tensional static loading. All of the cracks have equivalent distances between each other (Figure 1).

A list of symbols and parameters is compiled in Table 1 , as defined in the text and the following formulations.

The maximum number of layers in this analysis is four sublaminates; however, researchers are able to investigate for arbitrary lay-ups by handling their calculations following Ref. [11]. In addition, the thicknesses of these laminas

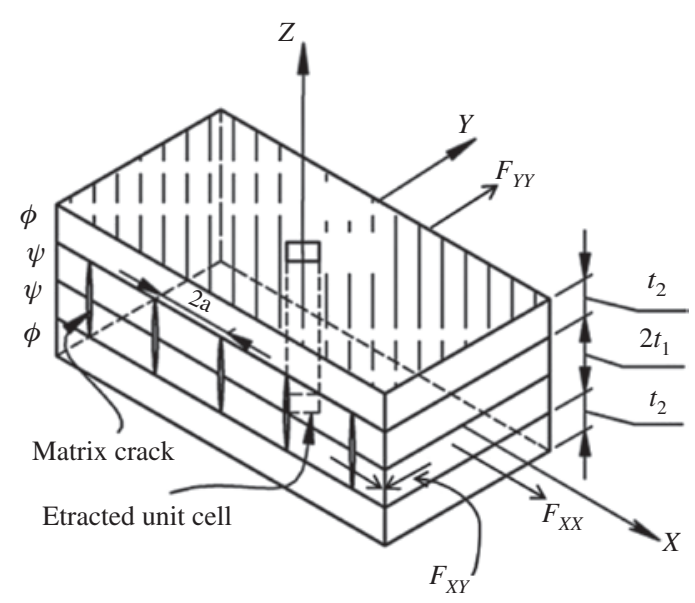

Figure 1: Geometry of a symmetric laminate containing cracks in the middle layers in a global coordinate system. 
Table 1: A list of parameters and their definitons in the text.

\begin{tabular}{|c|c|c|c|}
\hline Symbols & Definition & Symbols & Definition \\
\hline$\phi_{m}^{(2)}$ & The angle of layer (2) & $\eta(x)$ & An unknown function of $x$ \\
\hline$\psi_{n}^{(1)}$ & The angle of layer (1) & $2 a$ & The distance between the crack surfaces in a unit cell \\
\hline$F_{x x}$ & $X$-direction in-plane load (primary coordinate) & $C$ & The local compliance matrix \\
\hline$F_{X Y}$ & In-plane load in the $X Y$ plane (primary coordinate) & $U_{c}^{\prime}$ & Complementary energy due to perturbation stresses \\
\hline$F_{Y Y}$ & $Y$-direction in-plane load (primary coordinate) & $U^{u}$ & Complementary energy of the uncracked body \\
\hline$F_{x x}$ & $x$-Direction in-plane load (secondary coordinate) & $C^{\star}$ & The effective compliance matrix of the cracked body \\
\hline$F_{x y}$ & In-plane load in the $x y$ plane (secondary coordinate) & $W^{(i)}$ & The strain energy density due to perturbation stress \\
\hline$F_{y y}$ & $y$-Direction in-plane load (secondary coordinate) & $\varepsilon_{m n}^{p(i)}$ & The strain value of ply $(i)$ \\
\hline$t_{2}$ & The thickness of layer (2) & $\bar{C}^{(i)}$ & Compliance of the matrix unidirectional \\
\hline$t_{1}$ & The thickness of layer (1) & $\zeta$ & The ratio of $x$ to $t_{1}$ \\
\hline$\lambda$ & Dimensionless of quantity for thickness & $\rho$ & The ratio of $a$ to $t_{1}$ \\
\hline$h$ & The summation of thicknesses & $\alpha$ & The real part of the complex root \\
\hline$\theta(x)$ & An unknown function of $x$ & $\beta$ & The imaginary part of the complex root \\
\hline$\vartheta(x)$ & An unknown function of $x$ & $S^{*}(\rho)$ & The effective compliance matrix of the cracked unit cell \\
\hline$A_{t c}$ & The created damage area in micro cracking & $U_{c}^{b e}$ & The strain energy before micro cracking \\
\hline$U_{c}^{a f}$ & The strain energy after matrix cracking & $w$ & The width of laminate \\
\hline$d 2$ & The length of delaminated area in layer (2) - part 2 & $d 1$ & The length of delaminated area in layer (2) - part 1 \\
\hline$S_{2}$ & The compliance of outer lamina in delaminated composite & $U_{A \& C}$ & The strain energy after delamination for outer laminas \\
\hline$\delta$ & Dimensionless number for length of delaminated area & $V_{\rho-\delta}$ & The volume of intact region \\
\hline$A_{D}$ & The delaminated area & $\omega$ & The real and the positive root \\
\hline$U_{B}$ & The strain energy for the intact region & $G_{d}$ & The energy release rate of the delaminated unit cell \\
\hline$G_{m}$ & The energy release rate due to matrix cracking formation & $E_{A}$ & Axial Young's modulus in fiber direction \\
\hline$c$ & The crack density & $G_{T}$ & Transverse shear modulus \\
\hline$E_{T}$ & Transverse Young's modulus & $v_{A}$ & Associated axial Poisson's ratio \\
\hline$v_{T}$ & Associated transverse Poisson's ratio & $\sigma_{x x}^{00}$ & The applied stress in the $X$ direction \\
\hline$G_{A}$ & Axial shear modulus & $\sigma_{y y}^{00}$ & The applied stress in the $Y$ direction \\
\hline$\sigma_{x y}^{00}$ & The applied stress in the $X Y$ plane & $\sigma_{1}$ & The local stress in the 1 direction \\
\hline$\sigma_{2}$ & The local stress in the 2 direction & $\sigma_{3}$ & The local stress in the 3 direction \\
\hline
\end{tabular}

can be considered arbitrary. As shown in many papers [11, $12,24]$, these calculations are the same in estimating basic science; however, generally, their boundary conditions are different. The first step of calculations is assessing the stiffness degradation. For this purpose, it is needed to transfer the unit cell's coordinate to the new one. In fact, if it is assumed to continue calculations based on the project just like Hashin did in 1985 [10], changes should be made in its primary strategy. In order to have $90^{\circ}$ plies in the middle sublaminates, the rotation around $Z$ axis should be $\left[-\psi_{n}^{(1)}+90\right]$. Indeed, this method carries out the data to the previous calculations with cross-ply sublaminates. New lay-up for this composite can be displayed in the form of $[S / 90] s$. The term $S$ denotes $\left[\phi_{m}^{(2)}-\psi_{n}^{(1)}+90\right]$ in the general lay-up (Figure 2).

As illustrated, under in-plane loading, which can be arbitrary, these cracks are constructed. After the rotation, it is expected that the type of forces and their amounts would be altered. Lowercase letters represent new coordinate characteristics. Next, it is shown that in some rotations depending on their angles and their quantities, occasionally they would be reversed into compressive

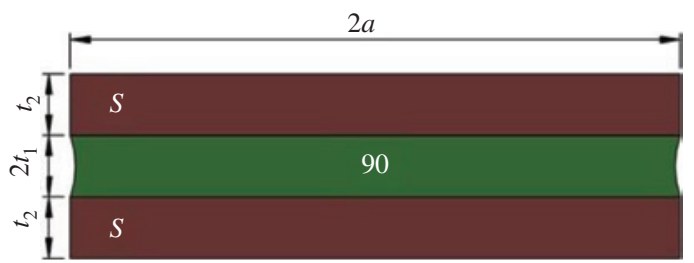

Depth $=1 \mathrm{~mm}$

Figure 2: Cracked laminate in the rotated coordinate.

loading. By these interpretations, we bring forward external loads for the primary coordinate as $F_{X X}, F_{X Y}, F_{Y Y}$ and for the secondary coordinate in $F_{x x}, \mathrm{~F}_{x y}, F_{y y}$. It is definitely seen that there are always some papers that have used some different types of loadings and lay-ups [34] (which work on stiffness reduction of off-axis laminates; neither induced delamination nor matrix cracking analysis for angle-ply laminates); however, in this research, it is tried to assess general loadings and stacking sequence.

Now, it is the time to explicate the composition of stress field in a cracked laminate. In a fragment, it can be expressed as an admissible stress field by a coincidence of 
the stresses in the uncracked material and some special unknown perturbation stresses that are due to the presence of cracks.

$$
\tilde{\sigma}_{m n}^{(i)}=\sigma_{m n}^{u(i)}+\sigma_{m n}^{p(i)},
$$

where $i=1,2$ specifies the number of laminas, and $u$ and $p$ denote uncracked and perturbation, respectively. Proportionate with what is shown in Figure $1, h=t_{1}+t_{2}$ and a dimensionless ratio $\lambda=\frac{t_{2}}{t_{1}}$ are defined. By virtue of symmetry, it is tried to analyze only half of the laminate. In this model, as is observable in Figure 1, there are several boundary and continuity conditions that can help arrive at a solution for the perturbation stresses. The following conditions have been used in calculations:

(a) There are no stresses on the out-plane surfaces (at $\left.z=\mp h, \tilde{\sigma}_{m z}^{(2)}=0\right)$.

(b) Using continuity conditions between interfaces, it can be seen that at $z= \pm t_{1}, \tilde{\sigma}_{m z}^{(1)}=\tilde{\sigma}_{m z}^{(2)}$.

(c) Free tractions on the crack surfaces denote that $\left(x= \pm a, \tilde{\sigma}_{m x}^{(1)}=0\right)$.

(d) The behavior of an uncracked laminate with a cracked laminate just like the work done by Vinogradov and Hashin [24] can be simulated; thus, the effects of local damages can be ignored.

Using the equilibrium equations and also balancing the membrane forces, some perturbation stresses that have impacts upon the stiffness degradation are achieved. Indeed, these amounts directly influence stiffness reduction. According to the steps that have been derived by Vinogradov and Hashin [24], the following can be written.

For lamina 1 (the middle lamina):

$$
\begin{aligned}
& \sigma_{x x}^{p(1)}=-\theta(x), \sigma_{x y}^{p(1)}=-\vartheta(x), \sigma_{y y}^{p(1)}=-\eta(x), \\
& \sigma_{x z}^{p(1)}=\theta^{\prime}(x) z, \sigma_{y z}^{p(1)}=\vartheta^{\prime}(x) z, \\
& \sigma_{z z}^{p(1)}=\theta^{\prime \prime}(x) \frac{\left(h t_{1}-z^{2}\right)}{2} .
\end{aligned}
$$

For lamina 2 (the outer ply):

$$
\begin{aligned}
& \sigma_{x x}^{p(2)}=\frac{1}{\lambda} \theta(x), \sigma_{x y}^{p(2)}=\frac{1}{\lambda} \vartheta(x), \sigma_{y y}^{p(2)}=\frac{1}{\lambda} \eta(x), \\
& \sigma_{x z}^{p(2)}=\frac{1}{\lambda} \theta^{\prime}(x)(h-z), \sigma_{y z}^{p(2)}=\frac{1}{\lambda} \vartheta^{\prime}(x)(h-z), \\
& \sigma_{z z}^{p(2)}=\frac{1}{\lambda} \theta^{\prime \prime}(x) \frac{(h-z)^{2}}{2},
\end{aligned}
$$

where all of these stresses are the perturbation stresses in two sublaminates. The applied boundary conditions are denoted by

$$
\theta( \pm a)=\sigma_{x x}^{u 1}, \theta^{\prime}( \pm a)=0, \vartheta( \pm a)=\sigma_{x v}^{u 1} .
$$

Note that these implicit results of boundary conditions will be used in calculating the final answers of ordinary differential equations of $(\theta, \vartheta, \eta)$, which are derived in the next step.

\subsection{Variational formulation}

Now, the principle of minimum complementary energy is going to be used for obtaining the perturbation stresses. For this purpose, the strain energy release rate must be calculated for transverse cracked laminates. It is very important to know about how the strain energy has to be derived in a model. For a loaded unit cell, the complementary energy functional can be defined as

$$
\tilde{U}_{C r}=\frac{1}{2} \int \tilde{\sigma}^{T} C \tilde{\sigma} d V .
$$

As it was mentioned in some papers like variational principles for generalized plane strain problems and their applications [32], the correct shape of the energy criterion should not be used in the form of strain energy; however, given that there is a linear elastic assumption, this equivalent is acceptable. Therefore, all equations that are considered have to be under this assumption. In the presence of cracks, for all elastic materials, Eq. (5) can be expressed in the following form:

$$
\tilde{U}_{C}=U^{u}+U_{C}^{\prime},
$$

where $U^{u}$ and $U_{c}^{\prime}$ are the strain energies for an uncracked body and due to perturbation stresses, respectively. As mentioned in Hashin's papers [10, 24, 35], the value of the complementary energy using admissible stresses can be derived from the principle of minimum complementary energy or, in another words, it can be written as

$$
\tilde{U}_{C}=\frac{1}{2} \bar{\sigma}^{T} C^{*} \bar{\sigma} V \leq \tilde{U}_{C r}=\frac{1}{2} \bar{\sigma}^{T} C^{u} \bar{\sigma} V+\frac{1}{2} \int \sigma^{p^{T}} C \sigma^{p} d V,
$$

where the terms $C^{*}$ and $C^{u}$ are the effective compliance matrix of cracked and uncracked bodies, respectively; $V$ is the volume of the cracked laminate; $\bar{\sigma}$ specifies the average in-plane stresses equal to applied stresses; and $\sigma^{p}$ denotes the perturbation stresses that are constructed by the presence of cracks. Actually, it is needed to minimize the right-hand side of Eq. (7) in order to derive the closest amount of the strain energy to a practical value. For this purpose, it has been suggested to minimize an integration that is produced by multiplying the perturbation 
energy density by other quantities, which are described in equations.

Until here, it has been attempted to put forward a model that is restricted by two cracks in the volume of a fragment with $2 a$ length and $|z| \leq h$. Due to the factors in Figure 1 and conditions that have been explained before, for half of a unit cell, it can be written that

$$
\begin{aligned}
W^{(i)}= & \frac{1}{2}\left(\sigma_{x x}^{p(i)} \varepsilon_{x x}^{p(i)}+2 \sigma_{x y}^{p(i)} \varepsilon_{x y}^{p(i)}+2 \sigma_{y z}^{p(i)} \varepsilon_{y z}^{p(i)}+2 \sigma_{x z}^{p(i)} \varepsilon_{x z}^{p(i)}+\sigma_{y y}^{p(i)} \varepsilon_{y y}^{p(i)}\right. \\
& \left.+\sigma_{z z}^{p(i)} \varepsilon_{z z}^{p(i)}\right),
\end{aligned}
$$

where $W^{(i)}$ and $\varepsilon_{m n}^{p(i)}$ are the strain energy density and strain value of the ply $(i)$. It is worth to mention that the strain energy density is a scalar function. The sum of integration of all sublaminates over the given volume provides the total strain energy of the body. To calculate the strain amount, or in another words $\left(\varepsilon_{m n}^{p(i)}=\bar{C}^{(i)} \sigma_{m n}^{p(i)}\right)$, we have to compute the compliance of the matrix unidirectional composite. For this purpose, as it was suggested by Vinogradov and Hashin [24], it is better to rotate the coordinate as was mentioned in Section 2.1. Embedding the expressions of perturbation stresses [Eqs. (2) and (3)] and stress energy densities in the perturbation strain energy, an integral calculus with constant coefficients is derived that assists researchers in computing the value of this expression to totalize with the strain energy of the uncracked body. The general terms of this integration is expressed here:

$$
U_{C}^{\prime}=\int_{-a}^{a} F\left(x, \theta, \theta^{\prime}, \theta^{\prime \prime}, \vartheta, \vartheta^{\prime}, \eta\right) d x .
$$

For solving this integration from the Euler-Lagrange method, it must be converted to dimensionless form with these changes $\left(\xi=\frac{x}{t_{1}}, \rho=\frac{a}{t_{1}}\right)$. The above equation can be illustrated as

$$
U_{C}^{\prime}=t_{1}^{2} \int_{-\rho}^{\rho} F\left(\zeta, \theta, \theta^{\prime}, \theta^{\prime \prime}, \vartheta, \vartheta^{\prime}, \eta\right) d \zeta
$$

where

$$
\begin{aligned}
& F\left(\zeta, \theta^{\prime}, \theta^{\prime \prime}, \vartheta, \vartheta^{\prime}, \eta\right) \\
& \quad=M_{1}^{a} \theta^{2}(\zeta)+M_{2}^{a} \vartheta^{2}(\zeta)+M_{3}^{a} \eta^{2}(\zeta)+M_{12}^{a} \theta(\zeta) \vartheta(\zeta) \\
& \quad+M_{13}^{a} \theta(\zeta) \eta(\zeta)+M_{23}^{a} \vartheta(\zeta) \eta(\zeta)+M_{1}^{b}\left(\theta^{\prime}(\zeta)\right)^{2} \\
& \quad+M_{2}^{b}\left(\vartheta^{\prime}(\zeta)\right)^{2}+M_{12}^{b} \theta^{\prime}(\zeta) \vartheta^{\prime}(\zeta)+M_{1}^{c} \theta^{\prime \prime}(\zeta) \theta(\zeta) \\
& \quad+M_{2}^{c} \theta^{\prime \prime}(\zeta) \vartheta(\zeta)+M_{3}^{c} \theta^{\prime \prime}(\zeta) \eta(\zeta)+M_{1}^{d}\left(\theta^{\prime \prime}(\zeta)\right)^{2},
\end{aligned}
$$

and the multipliers are the known amounts that are detailed in the Appendix. To compute the strain energy due to perturbation stresses, the unknown functions $[\theta(\zeta), \vartheta(\zeta), \eta(\zeta)]$ must be calculated from minimizing this integration. Because of three unknown functions, three Euler-Lagrange equations are needed, which are obtained from the below relations:

$$
\begin{gathered}
\frac{d^{2}}{d \zeta^{2}}\left(\frac{\partial F}{\partial \theta^{\prime \prime}}\right)-\frac{d}{d \zeta}\left(\frac{\partial F}{\partial \theta^{\prime}}\right)+\frac{\partial F}{\partial \theta}=0 . \\
\frac{d^{2}}{d \zeta^{2}}\left(\frac{\partial F}{\partial \vartheta^{\prime \prime}}\right)-\frac{d}{d \zeta}\left(\frac{\partial F}{\partial \vartheta^{\prime}}\right)+\frac{\partial F}{\partial \vartheta}=0 . \\
\frac{\partial F}{\partial \eta}=0 .
\end{gathered}
$$

Substituting the result of Eq. (14) into Eqs. (12) and (13), a new one is derived that is only a function of two variables.

$$
\eta=\left(\frac{M_{13}^{a}}{2 M_{3}^{a}} \theta+\frac{M_{3}^{c}}{2 M_{3}^{a}} \theta^{\prime \prime}\right)-\frac{M_{23}^{a}}{2 M_{3}^{a}},
$$

which helps us rewrite Eq. (11) as

$$
U_{C}^{\prime}=t_{1}^{2} \int_{-\rho}^{\rho} H\left(\zeta, \theta, \theta^{\prime}, \theta^{\prime \prime}, \vartheta, \vartheta^{\prime}\right) d \zeta .
$$

As shown in the above equation, one of the unknown functions is omitted easily $(\eta)$. The other independent unknown functions $(\theta, \vartheta)$ will be determined in the coupled linear differential equations when EulerLagrange equations are implemented for two times. Thus, it can be written as

$$
\begin{gathered}
T_{1}^{a} \theta^{\prime \prime \prime \prime}+T_{2}^{a} \theta^{\prime \prime}+T_{3}^{a} \theta+T_{4}^{a} \vartheta^{\prime \prime}+T_{5}^{a} \vartheta=0, \\
T_{4}^{a} \theta^{\prime \prime}+T_{5}^{a} \theta+T_{6}^{a} \vartheta^{\prime \prime}+T_{7}^{a} \vartheta=0,
\end{gathered}
$$

where $T_{i}^{a}$ are the constant coefficients that can be obtained according to the formulations based on the previous coefficients before implementing the variational approach. Depending on the boundary conditions, this coupled linear differential equation can be converted to the model that is suggested by Refs. [10, 23, 24].

\subsection{Compliance of cracked unit cell}

In order to solve coupled linear differential equations, there are several solutions that are suggested by commercial mathematical software. The authors prefer to utilize a more rational way, which is an analytical solution. Indeed, from the standpoint of mathematics, it is not logical that numerical methods are used instead of analytical ways when both solutions are available. 
For these grounds, let us consider a matrix that is deduced from the coupled linear differential Eqs. (17) and (18):

$$
L=\left[\begin{array}{cc}
T_{1}^{a} r^{4}+T_{2}^{a} r^{2} & T_{4}^{a} r^{2}+T_{5}^{a} \\
T_{4}^{a} r^{2}+T_{5}^{a} & T_{6}^{a} r^{2}+T_{7}^{a}
\end{array}\right] .
$$

As it is clear to have a specified solution for Eqs. (17) and (18), the determinate of the above relation [Eq. (19)] should be zero, and thus it can be written that

$$
|L|=0 \rightarrow L_{1} r^{6}+L_{2} r^{4}+L_{3} r^{2}+L_{4}=0,
$$

where

$$
\begin{gathered}
L_{1}=T_{1}^{a} T_{6}^{a}, L_{2}=T_{1}^{a} T_{7}^{a}+T_{2}^{a} T_{6}^{a}-\left(T_{4}^{a}\right)^{2}, \\
L_{3}=T_{2}^{a} T_{7}^{a}+T_{3}^{a} T_{6}^{a}-2 T_{4}^{a} T_{5}^{a}, L_{4}=T_{3}^{a} T_{7}^{a}-\left(T_{5}^{a}\right)^{2} .
\end{gathered}
$$

Due to the simplification of the equations, it is assumed that $k=r^{2}$. Depending on the boundary conditions, it can be observed that there might be two complex roots and one real positive root.

$$
k_{1}=\omega, k_{2,3}=\alpha \pm i \beta .
$$

This is not the right place to describe more points about the process of solution. With these interpretations, it is better to refer to differential equation books like that of Simmons [36]. It has indicators to solving these kinds of problems.

The general model of a system with these roots can be noted in the form of

$$
\begin{aligned}
\theta(\zeta)= & \mu_{1} C_{1} \cosh (s \zeta)+C_{2} \cosh (p \xi) \cos (q \zeta) \\
& +C_{3} \sinh (p \xi) \sin (q \zeta) . \\
\vartheta(\zeta) & =C_{1} \cosh (s \zeta) \Delta_{1} \cosh (p \zeta) \cos (q \zeta) \\
& +\Delta_{2} \sinh (p \zeta) \sin (q \zeta) .
\end{aligned}
$$

It is essential to note that this problem has symmetry of about $(x=0)$. Here, multipliers of $\theta(\zeta)$ and $\vartheta(\xi)$ have relations with each other. Given Eqs. (21) and (22) in calculations lead authors to express that

$$
\begin{gathered}
s=\sqrt{\omega}, \quad p, q=\frac{\sqrt{2}}{2} \sqrt{\sqrt{\alpha^{2}+\beta^{2}} \pm \alpha} . \\
\Delta_{1}=\left(C_{2} \mu_{2}+C_{3} \mu_{3}\right), \Delta_{2}=\left(C_{3} \mu_{2}-C_{2} \mu_{3}\right) . \\
\mu_{1}=-\frac{T_{5}^{a}+T_{4}^{a} \omega}{T_{3}^{a}+T_{2}^{a} \omega+T_{1}^{a} \omega^{2}} .
\end{gathered}
$$

$$
\begin{gathered}
\mu_{2}=-\frac{\left(T_{5}^{a}+T_{4}^{a} \alpha\right)\left(T_{7}^{a}+T_{6}^{a} \alpha\right)+T_{4}^{a} \beta^{2}}{\left(T_{7}^{a}+T_{6}^{a} \alpha\right)^{2}+\left(T_{6}^{a}\right)^{2} \beta^{2}} . \\
\mu_{3}=-\frac{\left(T_{4}^{a} T_{7}^{a}-T_{5}^{a} T_{6}^{a}\right) \beta}{\left(T_{7}^{a}+T_{6}^{a} \alpha\right)^{2}+\left(T_{6}^{a}\right)^{2} \beta^{2}} .
\end{gathered}
$$

The insertion of the expression in Eq. (4) helps us obtain the particular solutions of Eqs. (24) and (25). At first, it is needed to compute the constants $C_{(i)}$ and then there are precise answers for unknown functions $(\theta, \vartheta)$. This is a uniform solution with a less detailed description for calculating the stiffness degradation in comparison with Vinogradov and Hashin's work [24]. Briefly using these functions, an equation can be obtained for compliance of the cracked body, which helps authors to evaluate a true value for strain energy in a cracked body. The expression of compliance for a cracked body can be obtained as

$$
C^{*} \leq C^{0}+\frac{2 U_{C}^{\prime}}{\bar{\sigma}^{T} V \bar{\sigma}} .
$$

According to the obtained equation, it is noted that in the variational standpoint, the minimum amount of the perturbation strain energy in the unit cell has the principal impact on the effective compliance matrix $\left(C^{*}\right)$. Now, in this model, which is able to estimate the stiffness of a cracked body, it can be said that these calculations would not depend on the angles of sublaminates and it can be arbitrary.

\section{Energy release rate due to matrix cracking formation}

The first form of damage in laminates is often matrix cracking. By referring to performed studies $[6,8,31]$ and also according to the experimental results in cross-ply laminates, which are also mentioned in Ref. [31], the next matrix cracking is created in the middle layer. Among the performed studies, various methods of calculating the strain energy release rates have been suggested, which have infrastructural discrepancies. Indeed, as authors peruse these different methods carefully, they have been divided as (i) stress base [8] and (ii) stiffness base [31]. All of these methods have special advantages in their suitable positions. In this paper, it is tried to compute the strain energy release rate from the second technique. For the present state of matrix cracking, the strain energy can (before matrix cracking) be obtained from

$$
U_{c}^{b e}=\frac{1}{2} \bar{\sigma}^{T} S^{*}(\rho) \bar{\sigma} V .
$$




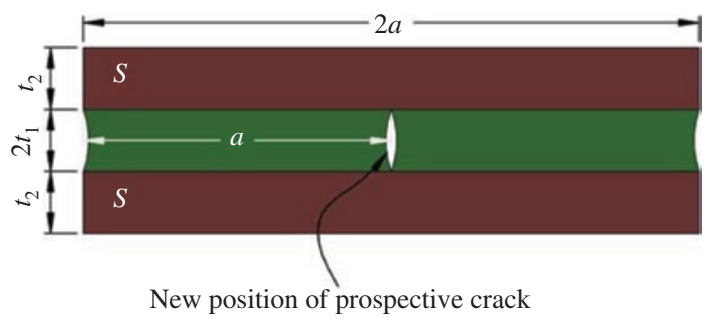

Figure 3: Unit cell after matrix cracking.

The strain energy after matrix cracking should be obtained by

$$
U_{c}^{a}=\frac{1}{4} \bar{\sigma}^{T} S^{*}\left(\frac{\rho}{2}\right) \bar{\sigma} V .
$$

It is important to mention that the parameter $A_{t c}$ is the created damage area and it must be evaluated from

$$
A_{t c}=2 t_{1} w
$$

and the energy release rate for this damage $\left(G_{m}\right)$ is finally obtained from

$$
G_{m}=\frac{\left(2 U_{c}^{a f}-U_{c}^{b e}\right)}{A_{t c}} .
$$

As shown in Figure 3, the initiation of matrix cracking in a unit cell is depicted. Indeed, two modes of laminate are investigated in these procedures, which should be divided after matrix cracking and before matrix cracking. It is conspicuous that after matrix cracking, the unit is separated into two segments. As a result, Eq. (33) defines an energy release rate after matrix cracking just for one segment.

This systematic approach is often used to detect an energy release rate based on the initial distance of cracks in a unit cell. In the next step, the other mode of damage is going to be investigated.

\section{Energy release rate due to induced delamination}

By initiation of delamination from tips of cracks, three regions are constructed as demonstrated in Figure 4. These regions are divided into intact and delaminated regions, respectively. In the intact region, the strain energy is calculated for the laminate containing transverse cracks with length of $2(\rho-\delta) t_{1}$. The delaminated region is also divided into two parts, which can be illustrated for outer plies and

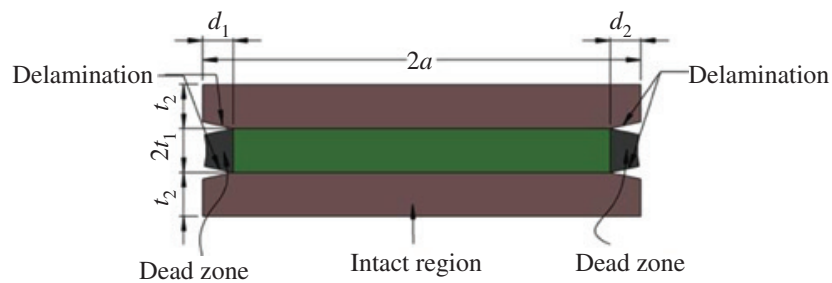

Figure 4: Different region of the delaminated unit cell.

the dead zone. It is only needed to compute the strain energy for outer laminas in a special volume, which is expressed below:

$$
U_{A \& C}=w\left(\int_{-a}^{-a+d 1} d x \int_{t_{1}}^{h} d z \tilde{\sigma}^{T} S_{2} \tilde{\sigma}+\int_{a-d 2}^{a} d x \int_{t_{1}}^{h} d z \tilde{\sigma}^{T} S_{2} \tilde{\sigma}\right),
$$

where $U_{A \& C}$ is the strain energy after delaminating of laminates for outer plies. In other words, integrating the strain energy density over a damaged area in an outer ply for sample of width $w$ gives the total strain energy in the damaged area. It is worth to mention that the middle sublaminate is the dead zone. Indeed, the stresses of other regions are zero and the middle sections can be removed from the sample with no effect. $\tilde{\sigma}_{i j}$ and $S_{2}$ are the admissible stress system and the compliance of the outer lamina, respectively. The stresses can be expressed as

$$
\tilde{\sigma}=\left[\begin{array}{lll}
\tilde{\sigma}_{x x} & \tilde{\sigma}_{y y} & \tilde{\sigma}_{x y}
\end{array}\right],
$$

and the in-plane stresses can be obtained from

$$
\begin{aligned}
& \tilde{\sigma}_{x x}=\sigma_{x x}^{u 1}-\phi(x)=\frac{(1+\lambda)}{\lambda} \sigma_{x x}^{00} . \\
& \tilde{\sigma}_{y y}=\sigma_{y y}^{u 1}-\psi(x)=\frac{(1+\lambda)}{\lambda} \sigma_{y y}^{00} . \\
& \tilde{\sigma}_{x y}=\sigma_{x y}^{u 1}-\eta(x)=\frac{(1+\lambda)}{\lambda} \sigma_{x y}^{00} .
\end{aligned}
$$

The boundary conditions in the outer plies require $\phi^{\prime}(x)=0, \psi^{\prime}(x)=0, \eta^{\prime}(x)=0$. Integrating these equations implies that the integrated functions are constants.

The strain energy release rate for the intact region should be obtained from

$$
U_{B}=\frac{1}{2} \bar{\sigma}^{T} S^{*}(\rho-\delta) \bar{\sigma} V_{\rho-\delta} .
$$

Note that Eq. (41) defines the strain energy for the intact region. For this purpose, it is worth to mention that when $\delta=0$, the initiation of delamination is calculable and this value depends only on the size of unit cells. $\delta$ has to be obtained from 


$$
\delta=\frac{d 1+d 2}{2 t_{1}},
$$

where $\tilde{\sigma}_{i j}$ and $S_{2}$ are the admissible stress system and the compliance of the outer lamina, respectively, and $V_{\rho-\delta}=2(\rho-\delta) t_{1} w$ is the volume of the intact region. Therefore, the energy release rate of the delaminated unit cell can be noticed as

$$
G_{d}=\frac{U_{B}+U_{A \& C}-U_{c}^{b e}}{A_{D}}
$$

where $A_{D}$ is the delaminated area. All of these calculations are based on a unit cell that is demonstrated in Figure 4.

\section{Results and discussion}

In this section, the energy release rates for diverse laminates with different stacking sequences and different thicknesses are considered under general loading conditions. These stresses have to be converted into the local coordinates. At first, the results of these calculations are compared with Nairn and Hu's results, and then it is tried to expand the results for variant lay-ups. Thereafter, the results of energy release rates are provided for distinct lay-ups.

\subsection{Cross-ply lay-ups}

Initially, it is better to introduce the same materials that had been used in Nairn and Hu's results [31].

$$
\begin{aligned}
& E_{A}=128 \mathrm{GPa}, G_{A}=4 \mathrm{GPa}, v_{A}=0.3 . \\
& E_{T}=7.2 \mathrm{GPa}, G_{T}=2.4 \mathrm{GPA}, v_{T}=0.5, t_{1}=t_{2}=0.14 \mathrm{~mm} .
\end{aligned}
$$

According to the utilized conditions by Ref. [33], the applied stresses are $\sigma_{x x}^{00}=100 \mathrm{MPa}, \sigma_{x y}^{00}=0, \sigma_{y y}^{00}=0$. If the presented Eqs. (35) and (42) are thoroughly investigated, the obtained results have incredible agreements to Ref. [33] for a $\left(\mathrm{O}_{2} / 90_{4}\right) s$ carbon fiber/epoxy as shown in Figure 5 , even though this process is much easier than theirs because of the ignorance of simplified additional equations.

Figure 5 illustrates the variation of energy release rates due to two forms of most possible damages with the increase of crack densities. It is shown that between 0 and 0.51 crack densities, the odds-on type of damage is formation of matrix cracking; however, from this critical point to the lowest distance between cracks, delamination must be observed. Generally, this critical point helps designers to know something more about the next possible damage. As shown in Figure 5, the size of the unit cell

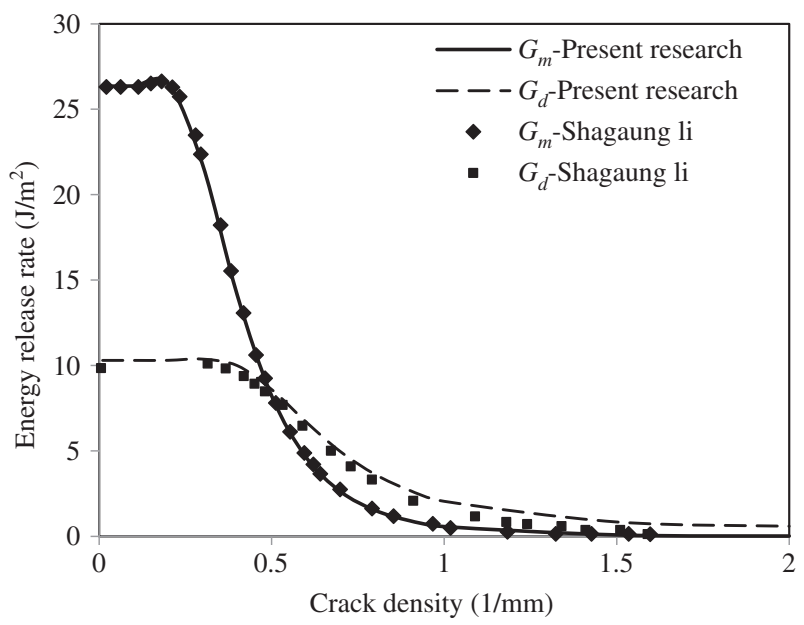

Figure 5: Comparison of the obtained energy release rate with Li's results for $\left[\mathrm{O}_{2} / 90_{4}\right] \mathrm{s}$ carbon/epoxy laminate under $\sigma_{1}=100 \mathrm{MPa}$ [33].

plays an essential role in the determination of the kind of damages. Moreover, this analogy proves the point that the new method of achieving energy release rate in the variational approach has the same precision that theirs have.

In the next attempts, the authors consider diverse cross-ply lay-ups of carbon fiber/epoxy, which are distinguished by their thicknesses. Likewise, the critical points are obtained easily, so that it is straightforward to estimate the effects of the number of cracked sublaminates in dislocation of critical points relative to crack densities. For this purpose, various types of fiber/epoxy cross-ply laminates are considered to report the influence of adding cracked laminas in Figures 6-8.

In all of these calculations, the considered stresses are $\sigma_{x x}^{00}=100 \mathrm{MPa}, \sigma_{x y}^{00}=0, \sigma_{y y}^{00}=0$; however, the authors

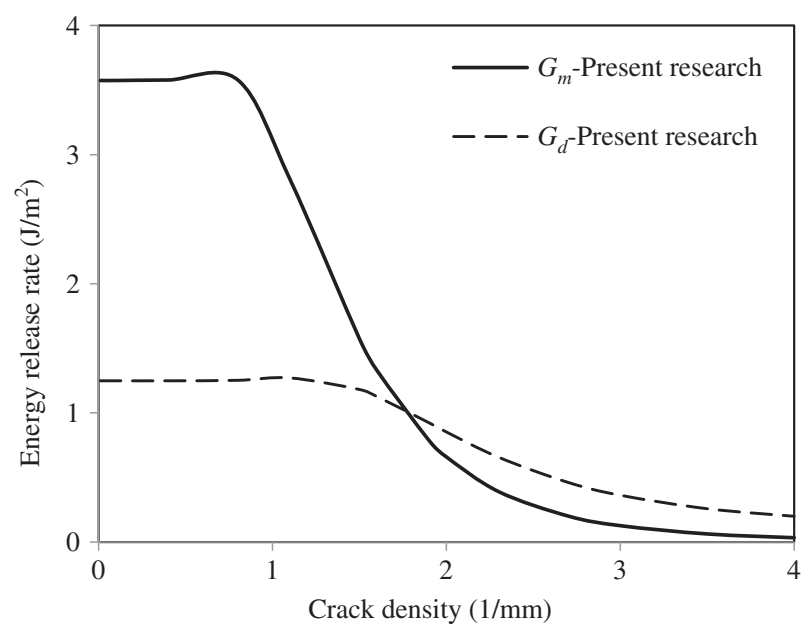

Figure 6: Crack density vs. energy release rate for [0/90]s carbon/ epoxy laminate under $\sigma_{1}=100 \mathrm{MPa}$. 


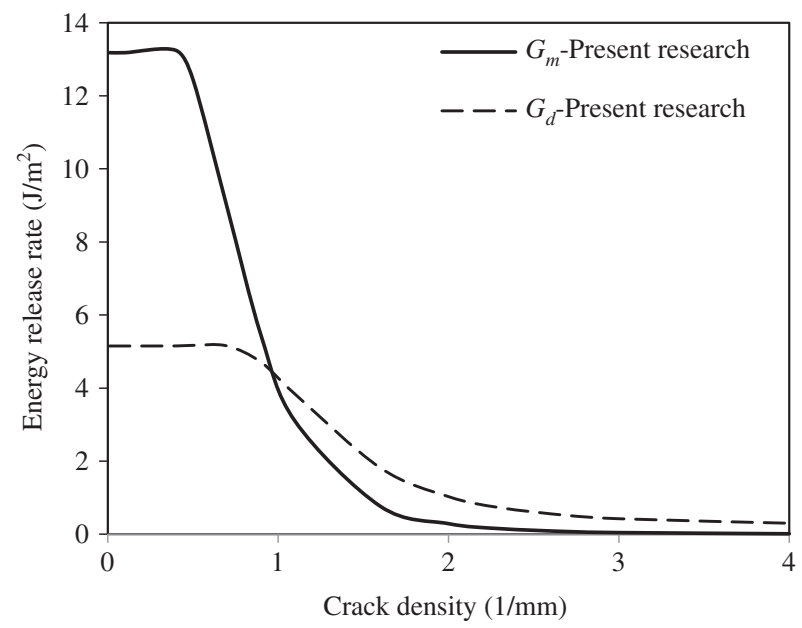

Figure 7: Crack density vs. energy release rate for $\left[0 / 90_{2}\right] \mathrm{s} \mathrm{carbon/}$ epoxy laminate under $\sigma_{1}=100 \mathrm{MPa}$.

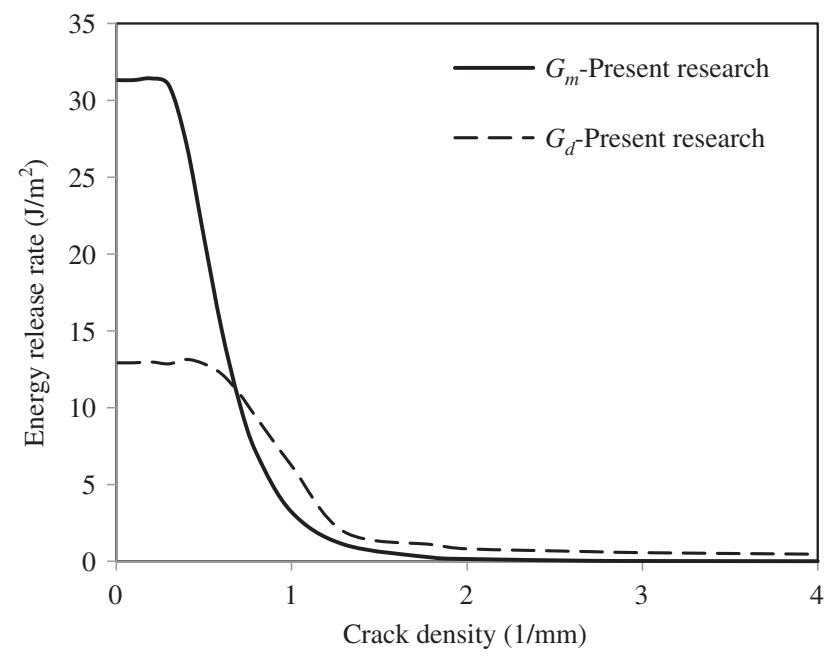

Figure 8: Crack density vs. energy release rate for $\left[0 / 90_{3}\right] s$ carbon/ epoxy laminate under $\sigma_{1}=100 \mathrm{MPa}$.

try to draw some graphs that have non-zero $\sigma_{x y}^{00}$ and $\sigma_{y y}^{00}$. Nevertheless, Figures 9-11 are provided as samples with other remote loadings. In these paradigms, the stresses are $\sigma_{x x}^{00}=100 \mathrm{MPa}, \sigma_{x y}^{00}=1 \mathrm{MPa}, \sigma_{y y}^{00}=10 \mathrm{MPa}$. As it is detectable in these cross-ply laminates, the increasing thicknesses of cracked sublaminates lead to regressive conditions, or, in another words, induced delamination is more prospective by declining the critical point relative to crack density.

\subsection{Angle-ply lay-ups}

Diverse angle-ply laminates are chosen to compute their energy release rates when their stresses in local

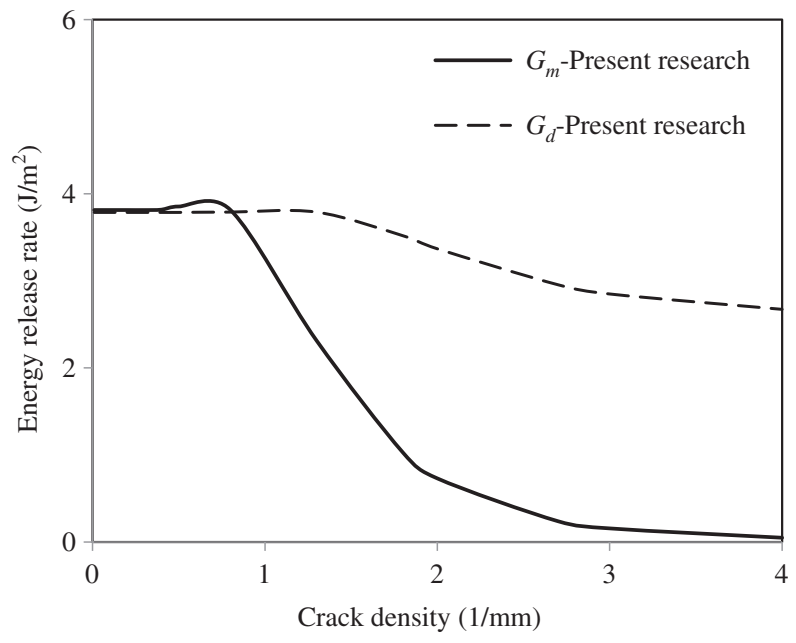

Figure 9: Crack density vs. energy release rate for [0/90]s carbon/ epoxy laminate under $\sigma_{1}=100 \mathrm{MPa}, \sigma_{12}=1 \mathrm{MPa}$ and $\sigma_{2}=10 \mathrm{MPa}$.

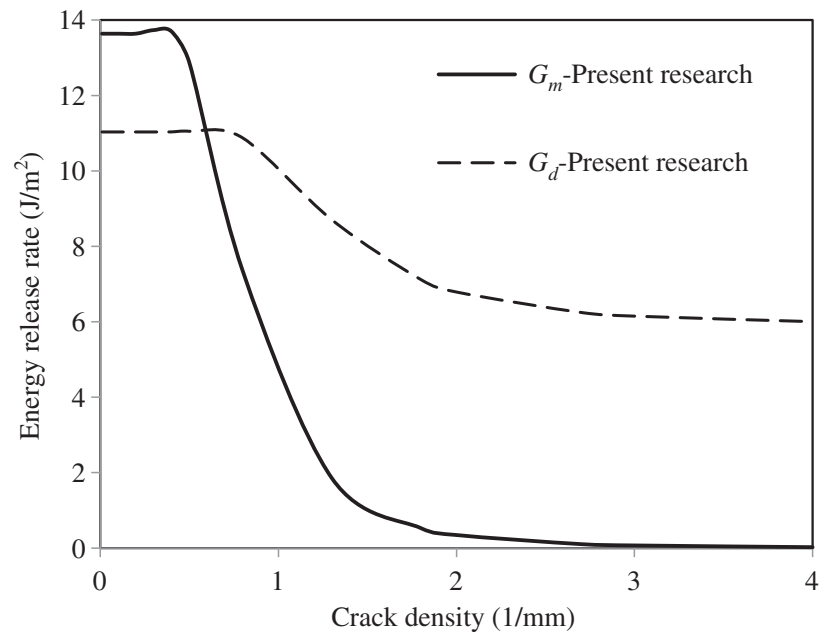

Figure 10: Crack density vs. energy release rate for $\left[0 / 90_{2}\right] \mathrm{s} \mathrm{carbon/}$ epoxy laminate under $\sigma_{1}=100 \mathrm{MPa}, \sigma_{12}=1 \mathrm{MPa}$ and $\sigma_{2}=10 \mathrm{MPa}$.

coordinates are the same as the precise amount that cross-ply laminates have. Indeed, the authors try to examine how differentiation of angles affects the energy release rate for induced delaminations and the next matrix cracking in a unit cell. According to these assessments, it is decided to provide Table 2, which introduces some essential factors about the stress and its angles for local coordinate.

Relatively straightforward calculations specify the point that in order to achieve angle-ply energy release rates, the expressions have to be investigated according to their local coordinates as Hosseini-Toudeshky et al. [8] did in their micromechanical approach. The results are drawn in diverse line charts, which are shown here. 


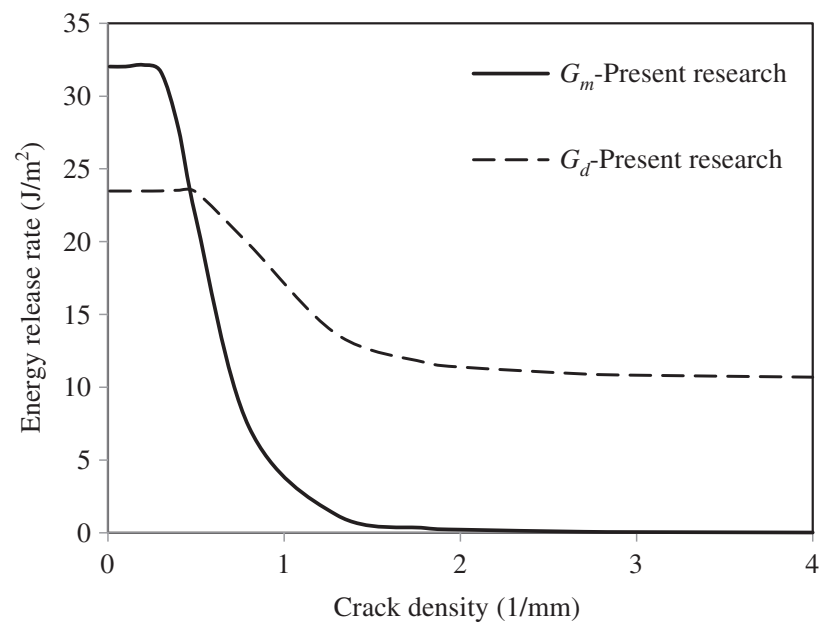

Figure 11: Crack density vs. energy release rate for $\left[0 / 90_{3}\right] \mathrm{s}$ carbon/ epoxy laminate under $\sigma_{1}=100 \mathrm{MPa}, \sigma_{12}=1 \mathrm{MPa}$ and $\sigma_{2}=10 \mathrm{MPa}$.

\subsection{1 $\left[45 /-45_{n}\right] s$ Angle-ply laminates}

Figures 12-14 show changes in the energy release rates of matrix cracking and induced delamination for $[45 /-45] \mathrm{s}$, $\left[40 /-45_{2}\right] s$ and $\left[40 /-45_{3}\right] s$. There is a modest increase in the energy release rate for matrix cracking and then it plunges sharply, although this steep decrease is not the same as the decline that is seen in the induced delamination. The critical points for Figures 12-14 are 0.81, 0.605 and 0.47, respectively. It should be underlined that the decreasing trend of critical points tends to be relative to the increasing number of thicknesses in the cracked laminas. In addition, according to the data shown, the amounts of energy release rate by increasing the number of cracked laminas are escalated.

\subsection{2 $[40 /-40]$ s Angle-ply laminates}

Figures 15-17 depict how inclinations for the energy release rates have changed for matrix cracking and induced delamination as well as Figures 12-14 illustrated. The most obvious changes, which are notable in comparison with the previous figures, are its measures for energy release rates. Indeed, whatever the stacking sequences of local coordinates $([S / 90] s)$ is closer to $[180 / 90] s$, the energy release rates see a slower change and vice versa. It can be clearly seen that the energy release rate for the next matrix cracking in [40/-40]s starts with $20.77 \mathrm{~J} / \mathrm{m}^{2}$; in contrast, this amount for [45/-45]s begins with $3.81 \mathrm{~J} / \mathrm{m}^{2}$.

Another distinguishing feature is their critical points. The variations of energy release rate versus crack density for matrix cracking and delamination intersect at 1.74 $(1 / \mathrm{mm}), 0.725(1 / \mathrm{mm})$ and $0.413(1 / \mathrm{mm})$ in Figures $16-18$. The trend of decreasing energy release rates is virtually the same as the other stacking sequences. As can be observed, some of these graphs are totally outnumbered by increasing the number of sublaminates.

\subsection{3 [30/-30 $]$ s Angle-ply laminates}

In this section, the widest difference can be seen in the amounts of energy release rates that Figures 18-20 demonstrate with the previous figures. As illustrated, the critical points are $1.96(1 / \mathrm{mm}), 0.925(1 / \mathrm{mm})$ and $0.524(1 / \mathrm{mm})$, respectively, for Figures 18-20.

This opportunity has been granted to express the standpoint that local stresses and also angles, when the middle layers are $90^{\circ}$, are determinative. In other words, for initiation of delamination and intralaminar matrix cracking, the key point is that how the global stresses would change and how these amounts affect the energy release rates according to their crack densities.

As the Euler-Lagrange equations are derived, it can be said that variational approaches can be acceptable if the orientation of fibers would be restricted in two layers and the intralaminar cracks would occur in the middle layer.

In Figure 21, the changes in critical densities for carbon/epoxy $\left[0 / 90_{n}\right] s,\left[45 /-45_{n}\right] s,\left[40 /-40_{n}\right] s,\left[30 /-30_{n}\right] s$ and $\left[15 /-15_{n}\right] s$ laminates are illustrated with respect to the number of middle cracked plies. The results demonstrate a rational trend according to the altering angles. In other words, whatever the number of cracked laminates are increased, the risk of delamination increases in proportion to the particular lay-up that is being investigated. According to previous studies [8], it can be proved, when the coordinates and the amounts of stresses are equal in

Table 2: Local and global loads of several laminates.

\begin{tabular}{lrrrrrrrr}
\hline No. & {$\left[\phi_{m}^{(2)} \psi_{n}^{(1)}\right] s$} & {$\left[\phi_{2} / \phi_{1}\right] s(\mathrm{a})$} & $\sigma_{1}(\mathrm{MPa})$ & $\sigma_{2}(\mathrm{MPa})$ & $\sigma_{12}(\mathrm{MPa})$ & $\sigma_{x x}^{00}(\mathrm{MPa})$ & $\sigma_{y y}^{00}(\mathrm{MPa})$ & $\sigma_{x y}^{00}(\mathrm{MPa})$ \\
\hline 1 & {$[45 /-45]$} & {$[180 / 90]$} & 100 & 10 & 1 & 56 & 54 & -45 \\
2 & {$[40 /-40]$} & {$[170 / 90]$} & 100 & 10 & 1 & 48.17 & 61.83 & -44.49 \\
3 & {$[30 /-30]$} & {$[150 / 90]$} & 100 & 10 & 1 & 33.366 & 76.634 & -39.47 \\
\hline
\end{tabular}




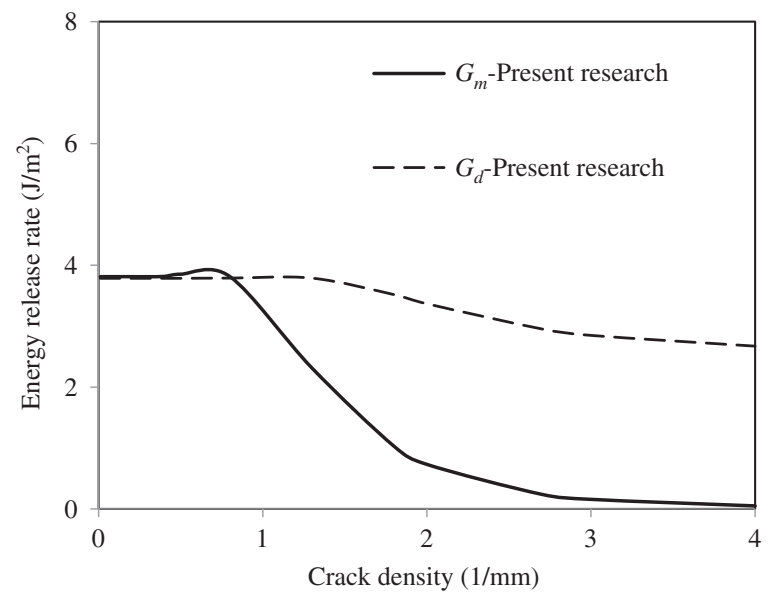

Figure 12: Crack density vs. energy release rate for [45/-45]s carbon/ epoxy laminate under $\sigma_{1}=100 \mathrm{MPa}, \sigma_{12}=1 \mathrm{MPa}$ and $\sigma_{2}=10 \mathrm{MPa}$.

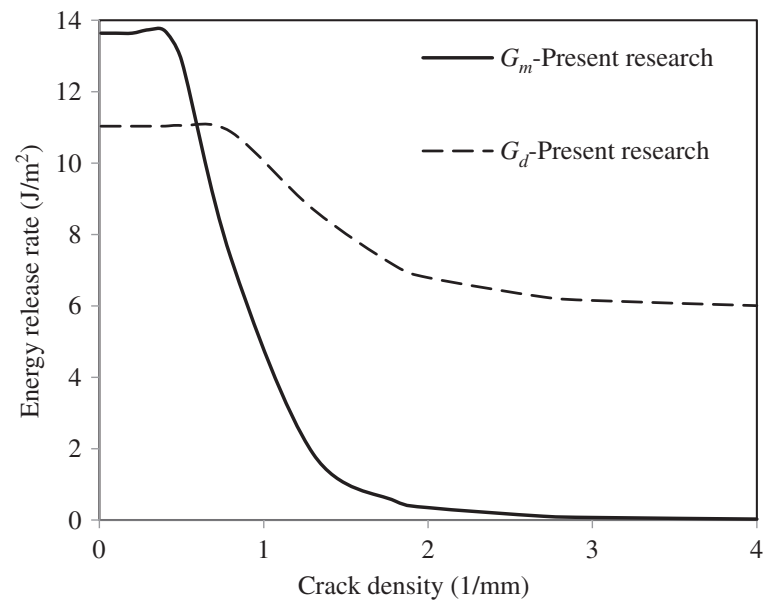

Figure 13: Crack density vs. energy release rate for [40/-45 $]$ s carbon/ epoxy laminate under $\sigma_{1}=100 \mathrm{MPa}, \sigma_{12}=1 \mathrm{MPa}$ and $\sigma_{2}=10 \mathrm{MPa}$.

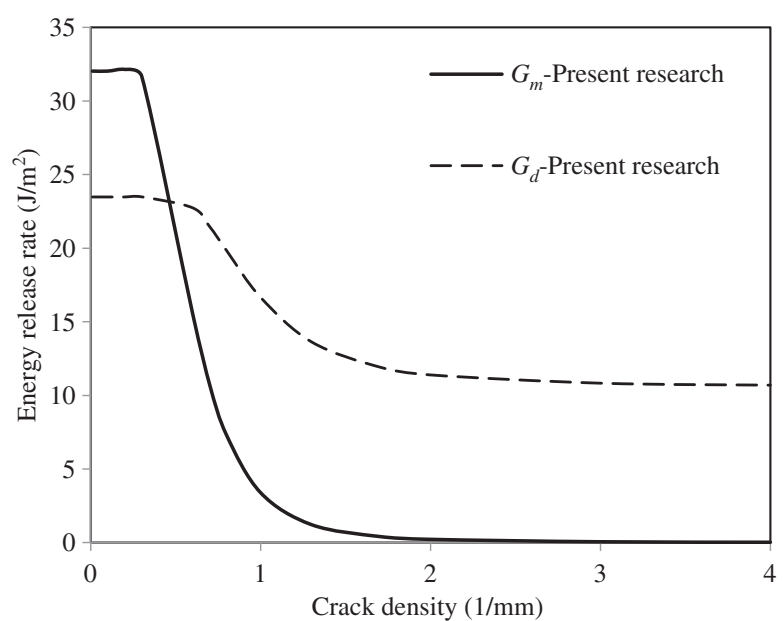

Figure 14: Crack density vs. energy release rate for [45/-45 $]$ s carbon/ epoxy laminate under $\sigma_{1}=100 \mathrm{MPa}, \sigma_{12}=1 \mathrm{MPa}$ and $\sigma_{2}=10 \mathrm{MPa}$.

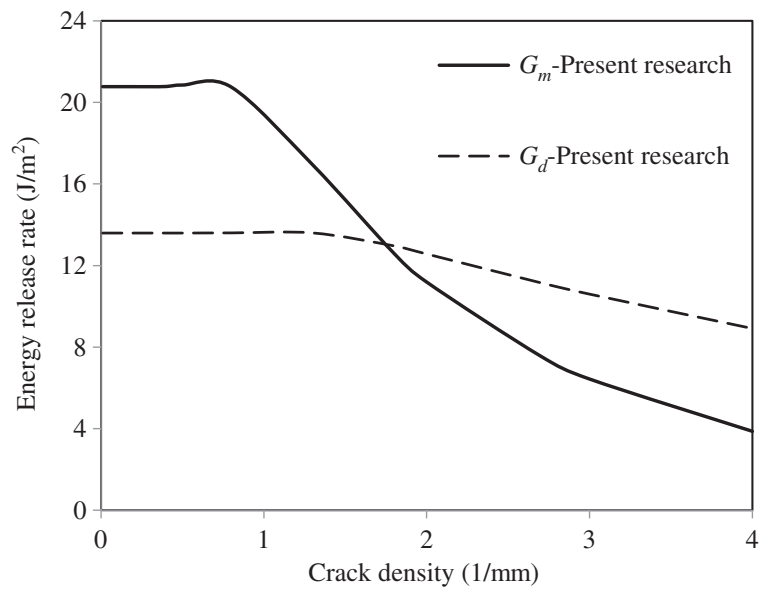

Figure 15: Crack density vs. energy release rate for [40/-40]s carbon/ epoxy laminate under $\sigma_{1}=100 \mathrm{MPa}, \sigma_{12}=1 \mathrm{MPa}$ and $\sigma_{2}=10 \mathrm{MPa}$.

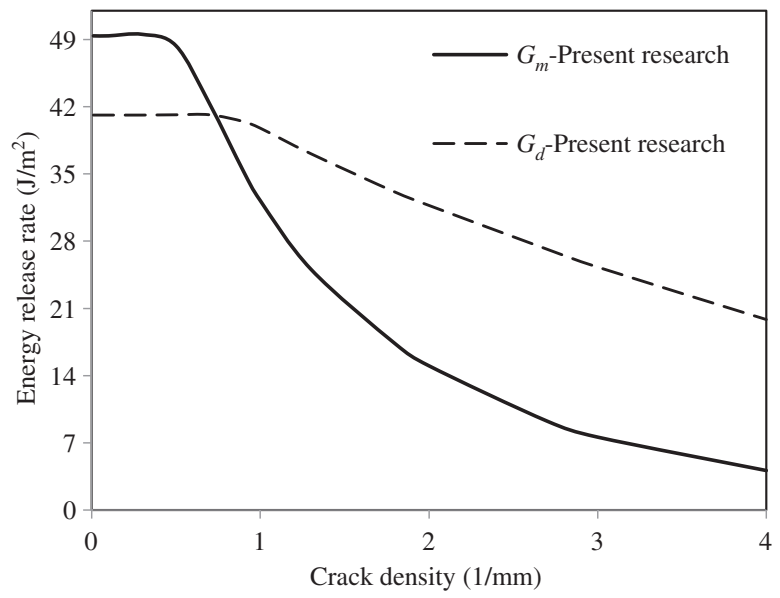

Figure 16: Crack density vs. energy release rate for $\left[40 /-40_{2}\right] \mathrm{s}$ carbon/ epoxy laminate under $\sigma_{1}=100 \mathrm{MPa}, \sigma_{12}=1 \mathrm{MPa}$ and $\sigma_{2}=10 \mathrm{MPa}$.

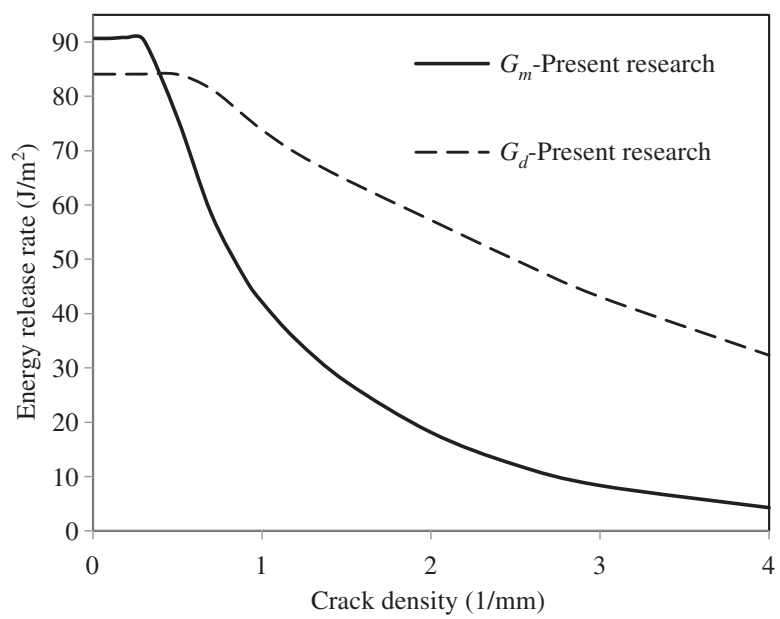

Figure 17: Crack density vs. energy release rate for $\left[40 /-40_{3}\right] \mathrm{s}$ carbon/ epoxy laminate under $\sigma_{1}=100 \mathrm{MPa}, \sigma_{12}=1 \mathrm{MPa}$ and $\sigma_{2}=10 \mathrm{MPa}$. 


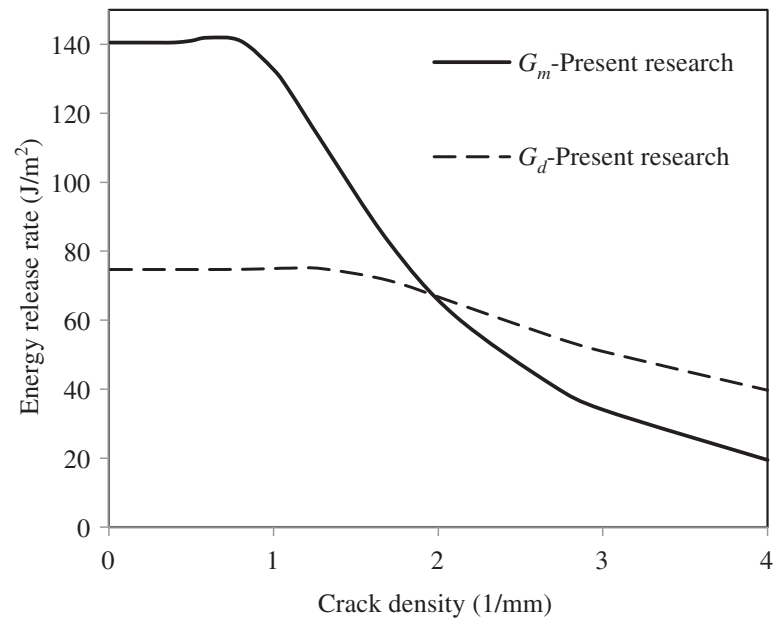

Figure 18: Crack density vs. energy release rate for [30/-30]s carbon/ epoxy laminate under $\sigma_{1}=100 \mathrm{MPa}, \sigma_{12}=1 \mathrm{MPa}$ and $\sigma_{2}=10 \mathrm{MPa}$.

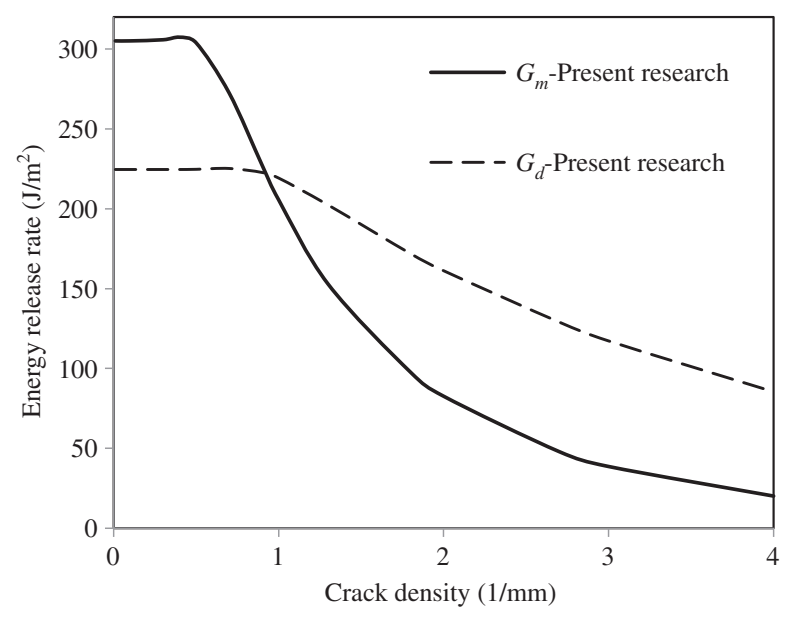

Figure 19: Crack density vs. energy release rate for $\left[30 /-30_{2}\right] s$ carbon/ epoxy laminate under $\sigma_{1}=100 \mathrm{MPa}, \sigma_{12}=1 \mathrm{MPa}$ and $\sigma_{2}=10 \mathrm{MPa}$.

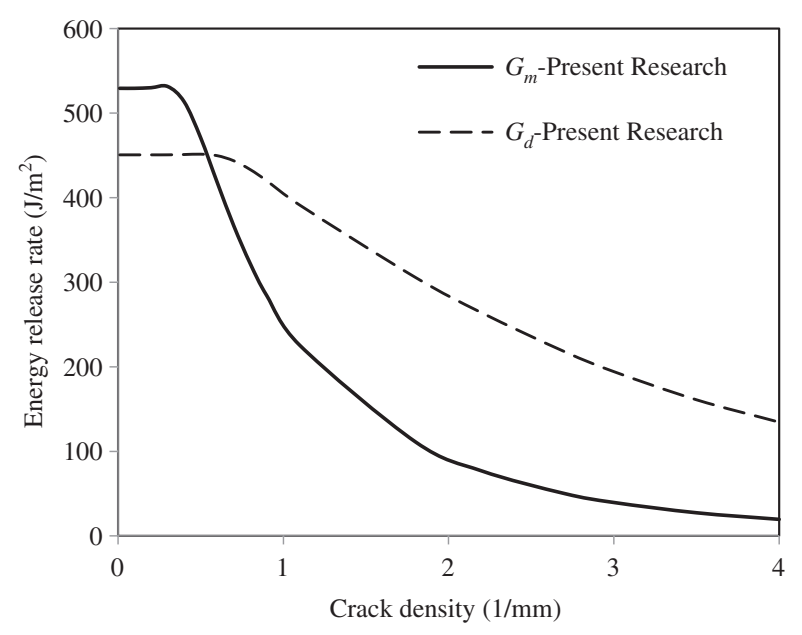

Figure 20: Crack density vs. energy release rate for $\left[30 /-30_{3}\right]$ s carbon/ epoxy laminate for under $\sigma_{1}=100 \mathrm{MPa}, \sigma_{12}=1 \mathrm{MPa}$ and $\sigma_{2}=10 \mathrm{MPa}$.

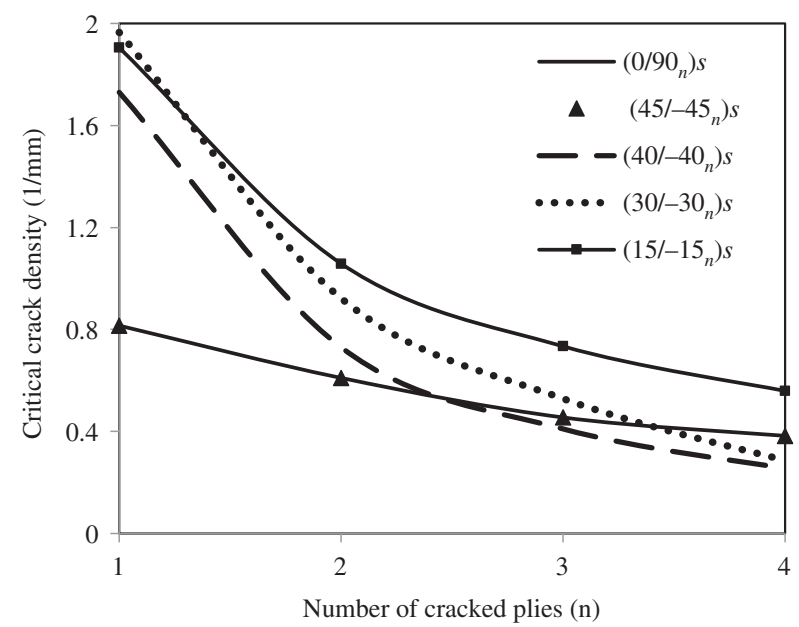

Figure 21: Number of middle cracked plies vs. critical crack densities for distinct carbon/epoxy laminates.

local calculations, that critical crack density points have broadly similar amounts.

Table 3 shows the critical energy release rates, critical crack densities, and energy release rates due to matrix cracking and induced delamination for a minute unit cell $(c=0.01) . G_{c}$ denotes the critical energy release rate, which represents the precise energy release rate for exceeding induced delamination in relation to matrix cracking as possible damages. Table 3 depicts that whenever stacking sequences in local coordinates get far from $\left[0 / 90_{n}\right] s$, the energy release rates increase dramatically. Furthermore, the amounts of critical crack densities are growing rapidly. In this table, it can be seen that almost all critical crack density values are decreasing by increasing the

Table 3: The critical energy release rates, critical crack densities and energy release rates due to induced delamination and matrix cracking for a unit cell with $c=0.01(1 / \mathrm{mm})$ under $\sigma_{1}=100 \mathrm{MPa}$, $\sigma_{12}=1 \mathrm{MPa}, \sigma_{2}=10 \mathrm{MPa}$.

\begin{tabular}{llrrrr}
\hline No. Lay-up & $\begin{array}{r}\text { Critical crack } \\
\text { density }(\mathbf{1} / \mathbf{m m})\end{array}$ & $\begin{array}{r}G_{c}\left(\mathrm{~J} / \mathrm{m}^{2}\right) \\
G_{m} / \mathrm{c}=\mathbf{0 . 0 1} \\
(\mathbf{1} / \mathbf{m m})\end{array}$ & $\begin{array}{r}G_{d} / \mathbf{c}=\mathbf{0 . 0 1} \\
(\mathbf{1} / \mathbf{m m})\end{array}$ \\
\hline 1 & {$[0 / 90] s$} & 0.81 & 3.79 & 3.813 & 3.785 \\
2 & {$\left[0 / 90_{2}\right] s$} & 0.61 & 11.06 & 13.636 & 11.032 \\
3 & {$\left[0 / 90_{3}\right] s$} & 0.47 & 23.48 & 32.028 & 23.477 \\
4 & {$[45 /-45] s$} & 0.81 & 3.79 & 3.813 & 3.785 \\
5 & {$\left[40 /-45_{2}\right] s$} & 0.61 & 11.06 & 13.636 & 11.032 \\
6 & {$\left[45 /-45_{3}\right] s$} & 0.47 & 23.48 & 32.028 & 23.477 \\
7 & {$[40 /-40] s$} & 1.73 & 13.088 & 20.77 & 13.59 \\
8 & {$\left[40 /-40_{2}\right] s$} & 0.73 & 41.19 & 49.382 & 41.133 \\
9 & {$\left[40 /-40_{3}\right] s$} & 0.411 & 84.19 & 90.66 & 84.076 \\
10 & {$[30 /-30] s$} & 1.975 & 67.222 & 140.508 & 74.702 \\
11 & {$\left[30 /-30_{2}\right] s$} & 0.923 & 222.014 & 305.058 & 224.559 \\
12 & {$\left[30 /-30_{3}\right] s$} & 0.529 & 451.260 & 512.55 & 450.651 \\
\hline
\end{tabular}


damaged area. In other words, decreasing from 0.81 to 0.47 in $\left[0 / 90_{n}\right] s$ shows that the possibility for matrix cracking is shrank by tripling the cracked sublaminates. This trend seems set to continue for other stacking sequences.

\section{Conclusion}

A variational technique for investigating initiation of matrix cracking and also delamination under general symmetric in-plane loading was provided. In the first step, a unit cell under general remote stress loading was considered, and then its stiffness reduction according to crack density was derived. In the following step, high standards were set for using general equations of energy release rates and then their values have been compared with the previous results.

For the first time, it was attempted to analyze the critical crack density of an angle-ply laminate. Heretofore, the energy release rates for cross-ply laminates had been calculated by some complex formulation for some special cases. However, this model covers all the laminates with different stacking sequences. The current variational approach is two-dimensional and does not explain the interlaminar normal stresses induced by a free edge. Therefore, it cannot be a good idea for growth of delamination.

Generally, when the sublaminate ( $S$ ) (for a laminate with stacking sequence like $\left[S / 90_{n}\right] S$ ) is adequately stiff and $n$ is not too small, the first phase of damage will be microcracking in the middle sublaminates. This trend will continue until the value of crack density reaches the critical crack density. It is clear that the more the cracked layers, the lower will be the critical crack density, and when the orientation of angles in the local coordinate gets far from the $\left[0 / 90_{n}\right] s$, the energy release rates are bigger than the ordinary situations.

Specifying critical distances between cracks are essential because these values must be considered to anticipate when a crack can constitute a real danger for a structure.

$$
\begin{aligned}
& M_{2}^{a}=\frac{1}{G_{A}} \frac{(1+\lambda)}{\lambda}+\left(\frac{1}{E_{T}}-\frac{1}{G_{A}}-\frac{1+2 v_{A}}{E_{A}}\right) \frac{\sin ^{2} 2 \gamma}{\lambda} . \\
& M_{3}^{a}=\frac{1}{E_{A}}+\frac{1}{\lambda E_{T}}-\left(\frac{1}{E_{T}}-\frac{1}{E_{A}}\right) \frac{\cos ^{2} \gamma}{\lambda} \\
& -\left(\frac{1}{E_{T}}-\frac{1}{G_{A}}-\frac{1+2 v_{A}}{E_{A}}\right) \frac{\sin ^{2} 2 \gamma}{4 \lambda} \text {. } \\
& M_{12}^{a}=\left(\frac{1}{E_{T}}-\frac{1}{E_{A}}\right) \frac{\sin 2 \gamma}{\lambda}+\left(\frac{1}{E_{T}}-\frac{1}{G_{A}}-\frac{1+2 v_{A}}{E_{A}}\right) \frac{\sin 4 \gamma}{2 \lambda} . \\
& M_{13}^{a}=\frac{-2 v_{A}}{E_{A}} \frac{(1+\lambda)}{\lambda}+\left(\frac{1}{E_{T}}-\frac{1}{G_{A}}-\frac{1+2 v_{A}}{E_{A}}\right) \frac{\sin ^{2} 2 \gamma}{2 \lambda} . \\
& M_{23}^{a}=\left(\frac{1}{E_{T}}-\frac{1}{E_{A}}\right) \frac{\sin 2 \gamma}{\lambda}-\left(\frac{1}{E_{T}}-\frac{1}{G_{A}}-\frac{1+2 v_{A}}{E_{A}}\right) \frac{\sin 4 \gamma}{2 \lambda} . \\
& M_{1}^{b}=\frac{1}{3}\left(\frac{1}{G_{T}}+\frac{\lambda}{G_{A}}\right)+\frac{\lambda}{3}\left(\frac{1}{G_{T}}-\frac{1}{G_{A}}\right) \cos ^{2} \gamma . \\
& M_{2}^{b}=\frac{1}{3}\left(\frac{\lambda}{G_{T}}+\frac{1}{G_{A}}\right)-\frac{\lambda}{3}\left(\frac{1}{G_{T}}-\frac{1}{G_{A}}\right) \cos ^{2} \gamma . \\
& M_{12}^{b}=\frac{\lambda}{3}\left(\frac{1}{G_{T}}-\frac{1}{G_{A}}\right) \sin 2 \gamma . \\
& M_{1}^{c}=\frac{v_{T}}{E_{T}}\left(\lambda+\frac{2}{3}\right)-\frac{v_{A} \lambda}{3 E_{A}}-\frac{\lambda}{3}\left(\frac{v_{T}}{E_{T}}-\frac{v_{A}}{E_{A}}\right) \cos ^{2} \gamma . \\
& M_{2}^{c}=\frac{v_{A}}{E_{A}}\left(\lambda+\frac{2}{3}\right)-\frac{v_{T} \lambda}{3 E_{T}}+\frac{\lambda}{3}\left(\frac{v_{T}}{E_{T}}-\frac{v_{A}}{E_{A}}\right) \cos ^{2} \gamma . \\
& M_{3}^{c}=-\frac{\lambda}{3}\left(\frac{v_{T}}{E_{T}}-\frac{v_{A}}{E_{A}}\right) \sin 2 \gamma . \\
& M_{1}^{d}=\frac{(1+\lambda)\left(3 \lambda^{2}+12 \lambda+8\right)}{60 E_{T}} .
\end{aligned}
$$

\section{References}

[1] Joffe R. Damage Accumulation and Stiffness Degradation in Composite Laminates, Thesis. Luleå University of Technology, 1999.

[2] Blázquez A, Mantič V, París F, McCartney NL. Solids Struct. 2008, 45, 1632-1662.

[3] Carlos G, Davila PPC, Rose, CA. J. Compos. Mater. 2005, 39, 323-345. 
[4] Camanho PP, Dávila CG, Pinho ST, Iannucci L, Robinson P. Compos. Part A Appl. Sci. Manuf. 2006, 37, 165-176.

[5] Hu S, Bark JS, Nairn JA. Compos. Sci. Technol. 1993, 47, 321-329.

[6] Nairn JA. J. Compos. Mater. 1989, 23, 1106-1129.

[7] Rebière J-L. Cogent Eng. 2016, 3, 1175060.

[8] Hosseini-Toudeshky H, Farrokhabadi A, Mohammadi B. Proc. Eng. 2011, 10, 236-241.

[9] Akula VMK, Garnich MR. Mech. Adv. Mater. Struct. 2014, 21, 737-748.

[10] Hashin Z. Mech. Mater. 1985, 4, 121-136.

[11] Hajikazemi M, Sadr MH. Int. J. Solids Struct. 2014, 51, 1483-1493.

[12] Hajikazemi M, Sadr MH. Int. J. Solids Struct. 2014, 51, 516-529.

[13] Wu X-F, Jenson RA, Zhao Y. Mech. Mater. 2014, 69, 195-203.

[14] Kashtalyan M, Soutis C. Int. J. Solids Struct. 2002, 39, 1515-1537.

[15] Chen H-S, Zhao Q-L, Gu Y, Yin Y-J, Fang D-N. Mech. Adv. Mater. Struct. 2013, 20, 564-570.

[16] Chen Z, Yan W. Mech. Mater. 2015, 91, 119-135.

[17] McCartney LN. J. Mech. Phys. Solids 1992, 40, 27-68.

[18] McCartney LN. In Local Mechanics Concepts for Composite Material Systems: IUTAM Symposium, Blacksburg, VA, 1991, Reddy JN, Reifsnider KL, Eds., Springer: Berlin, Heidelberg, 1992, pp. 251-282.

[19] McCartney LN. Compos. Sci. Technol. 2000, 60, 2255-2279.
[20] Adolfsson E, Gudmundson P. Int. J. Solids Struct. 1999, 36, 3131-3169.

[21] Okabe T, Imamura H, Sato Y, Higuchi R, Koyanagi J, Talreja R. Compos. Part A Appl. Sci. Manuf. 2015, 68, 81-89.

[22] Hashin Z. Eng. Fracture Mech. 1986, 25, 771-778.

[23] Hashin Z. Compos. Mater. 1987, 54, 872-879.

[24] Vinogradov V, Hashin Z. Compos. Sci. Technol. 2010, 70, 638-646.

[25] Zhang H, Minnetyan L. J. Reinforced Plastics Compos. 2006, 25, 1919-1938.

[26] Nairn JA, Hu S. Eng. Fract. Mech. 1992, 41, 203-221.

[27] McCartney LN. Compos. Sci. Technol. 1998, 58, 1069-1081.

[28] O’Brien TK. ASTM STP 876, 1985, 282-297.

[29] O’Brien TK. ASTM STP 1059, 1990, 9, 7-33.

[30] Dharani LR, Tang H. Int. J. Fract. 1990, 46, 123-140.

[31] Nairn JA, Hu S. Int. J. Fract. 1992, 57, 1-24.

[32] Li S, Lim S-H. Compos. Part A Appl. Sci. Manuf. 2005, 36, 353-365.

[33] Lim S-H, Li S. Compos. Part A Appl. Sci. Manuf. 2005, 36, 1467-1476.

[34] Huang ZQ, Zhou JC, He XQ, Liew KM. Int. J. Solids Struct. 2014, 51, 3669-3678.

[35] Hashin Z. Mater. Struct. 1983, 50, 481-505.

[36] Simmons G. Differential Equations with Applications and Historical Notes. McGraw-Hill, 1972. 